\title{
NMDA and muscarinic receptors of the nucleus accumbens have differential effects on taste memory formation
}

\author{
Leticia Ramírez-Lugo, Sergio Zavala-Vega, and Federico Bermúdez-Rattoni ${ }^{1}$ \\ Departamento de Neurociencias, Instituto de Fisiología Celular, Universidad Nacional Autónoma de México, \\ A.P. 70-253 México D.F., 04510, México
}

\begin{abstract}
Animals recognize a taste cue as aversive when it has been associated with post-ingestive malaise; this associative learning is known as conditioned taste aversion (CTA). When an animal consumes a new taste and no negative consequences follow, it becomes recognized as a safe signal, leading to an increase in its consumption in subsequent presentations (attenuation of neophobia, AN). It has been shown that the nucleus accumbens (NAcc) has an important role in taste learning. To elucidate the involvement of $N$-methyl-D-aspartate (NMDA) and muscarinic receptors in the NAcc during safe and aversive taste memory formation, we administrated bilateral infusions of DL-2-amino-5-phosphonopentanoic acid (APV) or scopolamine in the NAcc shell or core respectively. Our results showed that pre-training injections of APV in the NAcc core and shell disrupted aversive but not safe taste memory formation, whereas pre-training injections of scopolamine in the NAcc shell, but not core, disrupted both CTA and AN. These results suggest that muscarinic receptors seem to be necessary for processing taste stimuli for either safe or aversive taste memory, whereas NMDA receptors are only involved in the aversive taste memory trace formation.
\end{abstract}

The nucleus accumbens (NAcc) is a structure located in the rostrobasal forebrain and it is the major component of the ventral striatum. Three subterritories have been described for the NAcc; two caudal regions called core and shell, and the rostral pole (Zahm 2000). Several papers have related the functions of the NAcc with mechanisms of feeding (Kelley and Swanson 1997; Saul'skaya and Mikhailova 2003), motivation (Salamone 1996), reward (Berridge and Robinson 1998), and addiction (Bliss and Collingridge 1993; Everitt et al. 1999). Moreover, it has been suggested that gastric-related signals are transmitted to the NAcc via the hypothalamus, therefore, the NAcc could contribute to the integration of gastric vagal signals related to digestive processes (Mehendale et al. 2004).

When an animal consumes a new taste, it reduces its ingestion (neophobic response), and if there are no negative consequences afterwards, it becomes recognized as a safe signal, leading to a gradual increase of its consumption (attenuation of neophobia, AN). However, if the gustatory stimulus is paired with post-digestive malaise, the animal will recognize the taste as an aversive cue, thus developing conditioned taste aversion (CTA). Both aversive and safe memories depend on a neural representation of the taste, which is possibly stored in parallel in several brain regions. This neural representation has been called the Taste Memory Trace or TMT (Bermúdez-Rattoni 2004). It has been demonstrated that the insular cortex (IC), basolateral amygdala (BLA), parabrachial nucleus (PBN), and nucleus of the solitary tract (NTS) play important roles in taste memory formation (Yamamoto et al. 1994). Particularly, it has been described that the safe TMT depends on cortical muscarinic receptors' activity for consolidation, whereas the aversive TMT is at least partially dependent on the NMDA receptor activity (Gutierrez et al. 2003a).

Another structure that recently has been linked to taste

\section{'Corresponding author.}

E-mail fbermude@ifc.unam.mx; fax 011525556225607

Article and publication are at http://www.learnmem.org/cgi/doi/10.1101/ Im.103206. memory formation is the NAcc. The NAcc receives important inputs from structures involved in taste memories, like glutamatergic fibers from the BLA and IC (Kelley and Domesick 1982; Wright and Groenewegen 1996), as well as noradrenergic innervations from the NTS (Pennartz et al. 1994; Delfs et al. 1998). When saccharine has been associated with $\mathrm{LiCl}$, it produces a significant increase of acetylcholine (ACh) levels in the NAcc during its consumption (Mark et al. 1995). Additionally, it has been reported that intraoral novel saccharine infusion increases dopamine (DA) in the NAcc. However, the same stimulus previously associated with $\mathrm{LiCl}$, produced significant decreases in DA release (Mark et al. 1991). More recently Fenu and coworkers showed that a micro infusion of D1 antagonist (SCH 39166) into the NAcc shell impaired CTA learning (Fenu et al. 2001). Nevertheless, there is not enough information about the role of NMDA and muscarinic cholinergic receptors' functions in the NAcc during the TMT formation. Here we explore the role of NMDA and muscarinic receptors in safe and aversive taste learning by administrating bilateral infusions of APV (an antagonist of the NMDA receptors) and scopolamine (nonselective antagonist of muscarinic receptors), in the NAcc shell and core.

\section{Results}

Figure 1 shows an example of the NAcc cannulae placement. Histological analysis shows that in the majority of animals, the injection site was located in the NAcc shell (see Fig. 1A), or core (see Fig. 1B), respectively. Only the animals showing a correct placement of the cannulae were included in the statistical analysis. Thirteen animals were excluded from the analysis because the tips of their cannulas were misplaced.

\section{Pre-training APV or scopolamine disrupts short} and long aversive TMT

During the acquisition of CTA, all the animals drank the total amount of $10 \mathrm{~mL}$ presented. Figure $2 \mathrm{~B}$ shows the effects of muscarinic and NMDA receptor antagonists into the NAcc shell on 
A.

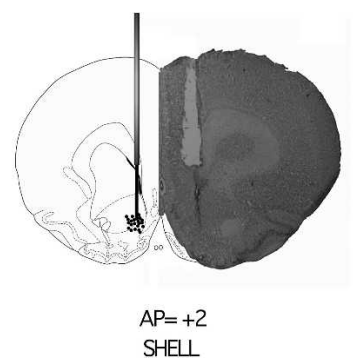

B.

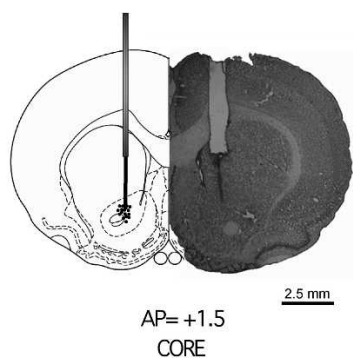

Figure 1. A photomicrograph of a coronal section of the rat brain stained with cresyl violet and a diagram of the cannulae location from the NAcc shell $(A, \mathrm{AP}=+2 \pm 0.3 \mathrm{~mm}$ from Bregma) or the NAcc core $(B, A P=+1.5 \pm 0.3 \mathrm{~mm}$ from Bregma) during microinjection. The points represent the localization of the cannulae.

both short-term memory (STM) and long-term memory (LTM) of conditioned taste aversion. No significant differences among groups were found in the water baseline. During the STM and LTM tests, significant differences in saccharine intake were found among groups $\left(F_{3,39}=8.865 ; F_{3,39}=6.605\right.$ respectively; $\left.P<0.01\right)$. Post hoc Fisher's test showed that both scopolamine and APV groups had a significantly higher saccharine consumption than the vehicle-injected and intact groups $(P<0.01)$.

Figure 2C shows the effects of muscarinic and NMDA receptor antagonists into the NAcc core. ANOVAs revealed significant differences between groups in saccharine consumption on the STM and LTM tests $\left(F_{2,20}=15.906 ; F_{2,20}=5.791\right.$, respectively, $P<0.01)$. Post hoc Fisher analysis revealed a significantly higher saccharine consumption in the APV group $(P<0.01)$. Thus, NAcc shell pre-training injections of APV and scopolamine impaired STM and LTM of CTA, while pre-training injection of APV, but not scopolamine into the NAcc core, impaired STM and LTM of CTA.

\section{Post-training injections of APV or scopolamine do not} block aversive TMT

APV or scopolamine injected immediately after the first presentation of saccharine during CTA training procedure yielded no significant differences among groups in saccharine consumption during the STM and LTM tests (see Fig. 3B,C). This was the case both when the antagonists were injected into the NAcc core $\left(F_{2,15}=0.633, \mathrm{NS}\right)$ or shell $\left(F_{2,15}=0.633, \mathrm{NS}\right)$.

Pre-training scopolamine, but not APV blocks safe TMT Figure 4B shows the effect of pre-training injection of APV, scopolamine or vehicle into the NAcc shell on neophobia (first presentation of saccharine taste) and on attenuation of neophobia (second presentation of saccharine), when no aversive consequences are induced (no lithium chloride injected after the first saccharine presentation, see Fig. 4A). ANOVA analyses showed that pre-training injection of APV or scopolamine did not affect the taste response to novelty $\left(F_{3,41}=0.5499\right.$, NS), while there were significant differences between groups $\left(F_{3,41}=17.390\right.$; $P<0.01)$ in attenuation of neophobia (AN, an increase in saccharine consumption in a subsequent presentation). Post hoc Fisher analysis revealed a significantly lower saccharine consumption during the AN test in the scopolamine group than in the vehicle group $(P<0.01)$, while the APV group did not differ significantly from the vehicle and intact groups. Thus, only the group injected with scopolamine into the NAcc shell failed to increase its saccharine consumption during the AN test, and therefore shows an abolished AN.

As can be seen in Figure 4C, no significant differences in neophobic response were seen between animals injected into the NAcc core with APV, scopolamine or ACSF injections
$\left(F_{2,14}=1.375\right.$; NS, see Fig. $\left.4 \mathrm{C}\right)$, which shows that APV or scopolamine injections before taste presentation do not affect taste perception. All animals show attenuation of neophobia (AN) during the second saccharine consumption. However, no significant differences among groups were found in saccharine consumption $\left(F_{2,14}=1.656\right.$; NS).

Additionally, no significant differences among groups were found in saccharine consumption during a third saccharine presentation, neither on the NAcc core-injected animals $\left(F_{2,13}=1.086\right.$, NS) nor on the NAcc shell-injected animals $\left(F_{3,41}=1.527, \mathrm{NS}\right)$ (data not shown).

\section{Discussion}

The present study was designed to investigate the effects of NMDA and muscarinic antagonists in the NAcc shell and core on the formation of taste memory. The NAcc has been widely reported to participate in appetitive reinforced learning tasks (Salamone et al. 2005). However, aversive and safe taste memory formation had not been included, expanding thus the role of the NAcc in learning processes.

In these experiments, we demonstrated that a pre-training injection of APV, on both the NAcc shell and core, impaired short-term and long-term aversive taste memory, without affect-

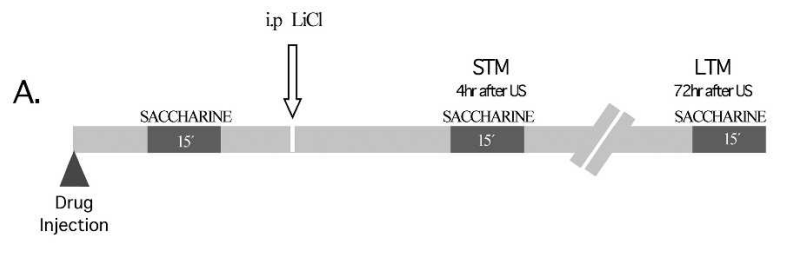

B.

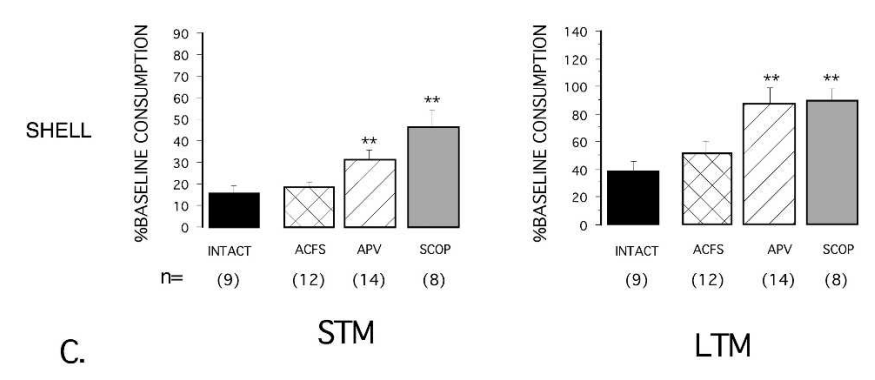

CORE
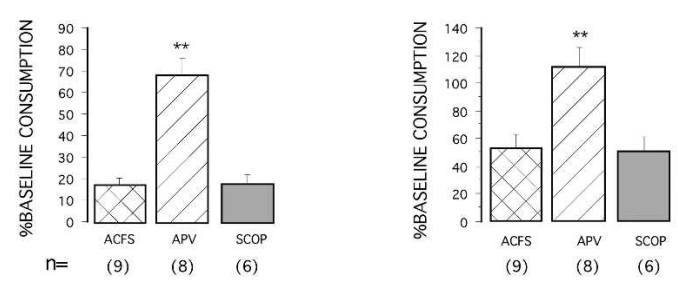

DRUG INJECTION BEFORE

Figure 2. (A) A representation of experimental protocols used to study the aversive TMT (pre-training injection) and percentage of baseline consumption (mean \pm SEM) on short and long-term test memory, for the NAcc shell (B) and the NAcc core (C). The reduction of saccharine consumption with respect to baseline intake was used as a measure of strength of aversion. The figure demonstrates the effects of APV and scopolamine (SCOP) microinjections 15 min before taste presentation of CTA. The "intact" group remained without treatment as naive control. $* \star P<0.01$, as compared to their vehicle controls (post hoc Fisher test), has significant differences from the other groups. 
A.

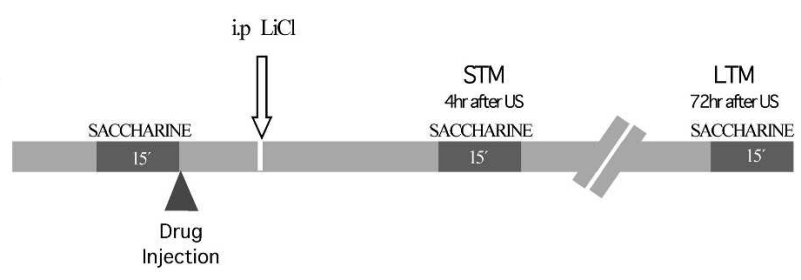

B.

C.
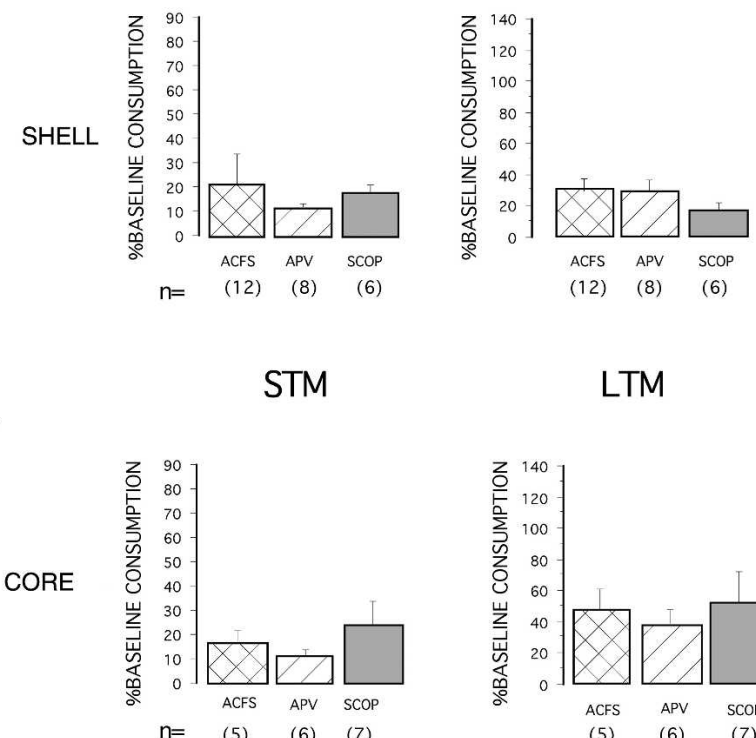

DRUG INJECTION AFTER

Figure 3. (A) A representation of experimental protocols used to study the aversive TMT (post-training injection) and percentage of baseline consumption (mean \pm SEM) on short and long-term test memory, for the NAcc shell $(B)$ and the NAcc core (C). The figure shows the effects of APV and scopolamine microinjections immediately after taste presentation of CTA. No significant differences among groups were found in saccharine consumption on the test of STM and LTM.

ing the safe taste memory formation. Conversely, post-training injections of APV did not have any effect on either task. These results show that the NMDA receptors in the NAcc shell and core have an important role in the aversive, but not safe TMT. Our results also showed the involvement of cholinergic transmission on the NAcc shell for the encoding of both safe and aversive TMT. Thus, pre-training injection of scopolamine on the NAcc shell disrupted both short-term and long-term aversive taste memory, and also prevented the attenuation of neophobia, whereas post-training injections of scopolamine had no effects in aversive memory formation. Furthermore, the present results showed that the neophobic response was not affected by APV or scopolamine, since the animals presented a clear neophobic response, ruling out unspecific effects on taste perception by the microinjections of NMDA or muscarinic antagonists.

\section{Glutamatergic activity}

The main result presented here is that the NMDA receptor in the NAcc shell and core has a differential role in taste aversive or safe memory formation. The NMDA receptor activity is necessary in both the NAcc shell and core for acquisition, but not for consolidation, of aversive taste memory. These results are in accordance with previous findings demonstrating that intra accumbens blockade of NMDA type glutamate receptors interfered with the acquisition process, but not with the consolidation of a passive avoidance task (Martinez et al. 2002). Moreover, we found that the APV produced stronger impairments in the formation of aversive STM, when injected in the NAcc core rather than in the NAcc shell, while its effects on LTM were similar in both sub nuclei. Accordingly, it has been proposed that NMDA receptor activation in the NAcc core may be an important mechanism through which stimulus-response associative learning occurs. Thus, injections of APV into the NAcc core, but not the shell, impaired response-reinforcement learning in the acquisition of a simple lever-press task to obtain food (Kelley et al. 1997). Conversely, for safe taste learning (AN), we found no effect of APV injection before taste presentation, suggesting that it does not affect the processing of the taste information required for safe taste memory formation. APV injections after taste presentation did not affect aversive taste learning, affecting only taste processing for aversive, but not safe, taste memory formation. These results support the proposed idea of the existence of two different memory traces generated by the consumption of a new taste, aversive and safe TMT (Gutierrez et al. 2003a; Bermúdez-Rattoni 2004), and suggest that the glutamatergic activity of the NAcc is involved only in the acquisition of aversive taste memory.

The taste and visceral information could reach the NAcc via two pathways: the NTS-parabrachial nucleus-central nucleus of the amygdala-ventral tegmental area-NAcc connection, and the insular cortex-BLA-NAcc pathway (McDonald 1991). It has been

A.

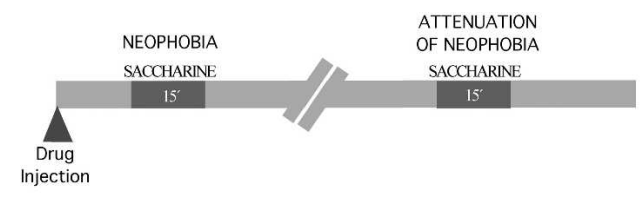

B.

SHELL
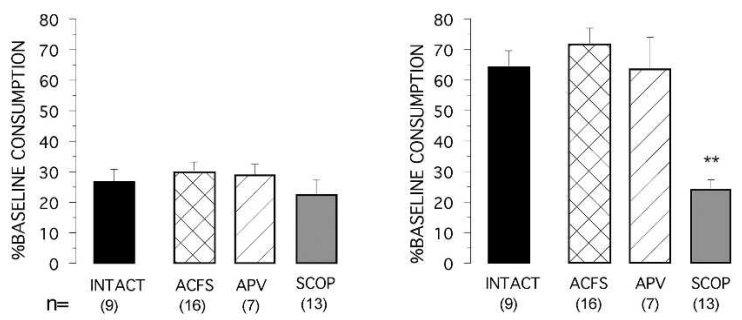

C.

NEOPHOBIA

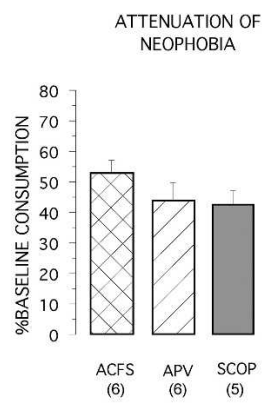

DRUG INJECTION BEFORE

Figure 4. (A) A representation of experimental protocols used for studying the safe TMT. The effect of injection of muscarinic receptor antagonist, scopolamine (SCOP), NMDA receptor antagonist (APV), and vehicle (ACFS) microinjected bilaterally into the NAcc shell $(B)$ and the NAcc core (C) 15 min before presentation of saccharine on $d 5$. The Neophobia and AN are expressed in percentage of baseline consumption. ${ }^{* *} P<0.01$, as compared to their vehicle controls (post hoc Fisher test), significantly differ from the other groups. 
considered that the central nucleus is the main output nucleus of the amygdala, mainly by GABAergic efferents to midbrain autonomic sites (Swanson and Petrovich 1998). However, the central nucleus of amygdala connection to the NAcc has been less studied. Alternatively, the BLA sends a dense glutamatergic projection to the NAcc (Kelley and Domesick 1982; Brog et al. 1993; Wright and Groenewegen 1996). It has been shown that the BLA and the NAcc interact during the formation and expression of stimulus-reward associations (Everitt et al. 1991). In this regard, for CTA a dramatic increase in glutamate release in the BLA was observed after an injection of $\mathrm{LiCl}$, suggesting that glutamate activity in the BLA signals the aversive input (Miranda et al. 2002). Therefore, given the participation of BLA in the processing of the aversive stimulus, it could be possible that the connection between BLA and NAcc plays a central role in relating the aversive taste memory trace with the information of the malaiseinducing agent. Even though our study cannot answer this issue, we propose that this mechanism would be implicated in aversive taste learning. Supporting this hypothesis, there is evidence of the participation of the NAcc in aversive learning (Pezze et al. 2001; Young 2004), and it is also suggested that NAcc dopamine is involved in aspects of sensory-motor functions that could be involved in aversive motivation (Salamone 1994). In this regard, neurochemical experiments have shown that both, naturally aversive and conditionally aversive stimulus, produce significant increments of dopamine release in the NAcc (Young et al. 1993). Complementarily, the insular cortex is highly involved in aversive taste learning (Bermúdez-Rattoni 2004) and since the BLA and the NAcc are important targets of agranular insular cortex efferent fibers (Ohara et al. 2003; Jasmin et al. 2004), and the innervations from the BLA coincides with projections from the dorsal agranular insular cortex into the NAcc core (Wright and Groenewegen 1996), it has been proposed, in an aversive learning situation, that at least some BLA-fugal fibers exert an inhibitory influence at the NAcc core level in the fibers coming from insular cortex (Louilot and Besson 2000). This might be the reason why we found stronger effects in the NAcc core compared to the NAcc shell. Therefore, a possible mechanism involved in the formation of aversive taste memory is the communication between the NAcc core, IC, and BLA, however this does not rule out the possibility that there could be other brain systems participating in the aversive taste memory along with the NAcc circuit proposed here.

It has been demonstrated that both subnuclei (shell and core) communicate through collateral neurons (Meredith 1999); the possibility exists that the effect observed in the NAcc shell after APV injection could be due to an interruption in the communication between them. It is important to examine the glutamatergic projection to the NAcc shell from the amygdala. Probably the effect observed in the NAcc shell is a consequence of blocking this projection. It also remains to be demonstrated whether there is an associative mechanism in the NAcc itself or if it modulates another structure where the aversive signals arrive from the BLA creating the aversive TMT. Since the coordinates for both subnuclei of the NAcc are very close to each other and we use different volumes, there is a possibility that the infusion of the drug in one subnuclei might produce some leakage to the other. Nevertheless, while we cannot rule out completely this possibility, we do believe that this did not happen since the effects of APV seem to be larger when applied in the core than in shell, and for scopolamine the effects were in the shell but not in the core (see below).

It is noteworthy that although the majority of reports of the NAcc on learning indicate its role in goal directed tasks with positive reinforcements (Everitt et al. 1999), we found that the effects of NMDA receptor antagonist are exclusively in the aver- sive, but not in the safe taste memory formation. Nevertheless, we can not rule out the possibility of other neurotransmitter systems participating in safe taste learning processing in this structure, like the muscarinic ACh receptors (see below).

An interesting fact about the present results is that the NMDA receptor blockade in the NAcc affects both STM and LTM of CTA. This implies that the pharmacological treatment avoids the formation of an aversive taste memory completely, not merely memory consolidation (Martinez et al. 2002). This supports the proposed idea that NMDA receptor activation is necessary for the formation of the aversive TMT, which eventually might form an aversive STM and LTM. Therefore the NAcc, through the NMDA receptors, is involved in the aversive taste memory formation; these results are in accord with previous findings that demonstrate the role of the NAcc in others' aversive learning such as a passive avoidance task (Martinez et al. 2002; De Leonibus et al. 2003), aversively conditioned contextual stimuli (Pezze et al. 2001), and aversive olfactory learning in humans (Gottfried et al. 2002).

\section{Cholinergic activity}

NAcc cholinergic activity has previously been reported to be related with conditioned taste aversion learning (Mark et al. 1995). As noted, there is a significant increment of ACh release in the NAcc after the presentation of a conditioned aversive taste (Mark et al. 1995). In agreement with these results, we found that injections in the NAcc shell of scopolamine before, but not after taste presentation abolished STM and LTM of CTA, supporting the role of ACh activity in the NAcc on taste memory processing. We did not find any effects when the drugs were administered after the CS, ruling out any unspecific effects on the incoming US signal. However, as pre-training injection of scopolamine also disrupts $\mathrm{AN}$, these results suggest that the activation of muscarinic receptors in the NAcc shell is necessary for normal learning about a taste stimulus. It should be pointed out that the injection of scopolamine did not affect taste perception, since the neophobic response to saccharine remained intact, regardless of the consequences of taste, i.e., safe or aversive TMT. These results support that CTA and AN initially share a similar component of the neural taste representation (Gutierrez et al. 2003b), which would be a common mechanism for both taste memory traces, through the activation of muscarinic receptors. Unlike APV, scopolamine injections either before or after taste in the NAcc core do not have any effect in aversive taste memory; these results indicate a differential role of cholinergic system in the NAcc on taste memory formation. It seems that the NAcc shell cholinergic activity is mainly involved in the formation of the TMT that can be later associated with either aversive or safe consequences. Although, it remains to be demonstrated whether the NAcc interneurons themselves could be participating in the associative process of taste presentation and its consequences to establish longterm memory for taste (either aversive or safe), since it has been demonstrated that the striatal cholinergic interneurons have an important role in triggering the intracellular mechanism that participates in synaptic plasticity, like corticostriatal facilitation (Centonze et al. 1999, 2003).

The cholinergic activity within the NAcc comes from the cholinergic interneurons (Pennartz et al. 1994) that are present in both shell and core subdivisions (Bolam et al. 1984; Meredith et al. 1989). ChAT-inmunoreactive neurons are nearly three times denser in the caudal medial shell than in the core (Meredith et al. 1989). It has been reported that GABAergic output from the NAcc can be regulated positively by nicotinic ACh receptors, and negatively by muscarinic ACh receptors (de Rover et al. 2002). A possible mechanism for the participation of muscarinic receptor in the NAcc shell in taste learning is through its modulation to the 
nucleus basalis magnocellularis (NBM) and in turn with its cholinergic projections to the insular cortex (Miranda and BermúdezRattoni 1999). It has been demonstrated that the NAcc shell can regulate $\mathrm{ACh}$ release in prefrontal cortex via its modulation to NBM (Neigh-McCandless et al. 2002). It has been suggested that the NAcc regulates the excitability of the NBM cortical cholinergic projections, by the GABAergic projection from the NAcc shell (Zahm and Heimer 1993; Neigh et al. 2004). Thus, inactivation of muscarinic ACh receptors in the NAcc might lead to an increase in GABAergic output projecting to NBM, generating a change in the levels of ACh in the cortex. It is well demonstrated that the cholinergic activity in IC has an important role in taste memory formation (Naor and Dudai 1996; Ferreira et al. 2002; Ramírez-Lugo et al. 2003). It is possible that the intra NAcc cholinergic activity modulates the taste inputs to the IC or to the amygdala through its connectivity with regions involved in taste memory formation.

\section{Conclusions}

These results provide the first evidence of the NAcc core and shell participation in the formation of aversive, but not safe TMT through their NMDA receptors. In addition, the results also showed the participation of the NAcc shell in the formation of both, aversive and safe TMT through its muscarinic receptors. Thus, the results presented here support the existence of two memory traces triggered by taste consumption, one of which is ruled out afterwards depending on the gastric consequences of the taste, which determines the formation of an aversive or safe gustatory memory. In this regard, the results showed that NMDA receptor activity of the NAcc core and shell seems to be involved in the formation of aversive taste memory trace, probably through their glutamatergic projections coming from the BLA, whereas muscarinic receptors' activity of the NAcc shell are involved in the initial formation of both taste memory traces, which can be later supported with either aversive or safe consequences.

\section{Materials and Methods}

\section{Animals}

Male Wistar rats $(N=185)$, with a weight between 280 and $320 \mathrm{~g}$ at the time of surgery were used. They were caged individually and kept in a 12-h light/12-h dark cycle phase. All behavioral manipulations were performed in the light cycle phase. Rats received lab chow throughout the experiment. Experiments were performed in accordance with the Rules in Health Matters (Ministry of Health, Mexico) and with approval of the local Animal Care Committee.

\section{Surgery}

The animals were implanted bilaterally with 12-mm 23-gauge stainless steel cannulae under anesthesia (sodium pentobarbital, $40 \mathrm{mg} / \mathrm{kg}$, i.p.) using standard stereotaxic procedures. The tips of the cannulae were aimed $2.5 \mathrm{~mm}$ above either the NAcc shell (anteroposterior, $+2 \mathrm{~mm}$; lateral, \pm 1 ; ventral, $-5.3 \mathrm{~mm}$; from Bregma); or the NAcc core (anteroposterior, $+1.5 \mathrm{~mm}$; lateral, \pm 1.9 ; ventral, $-4.7 \mathrm{~mm}$; from Bregma) (Paxinos and Watson 1998). The cannulae were fixed to the skull with dental acrylic cement and anchored with two surgical screws placed in the skull. Stylets were inserted into the guide cannulae to prevent clogging. After surgery, the animals had food and water ad libitum during four days, and the following day they were deprived of water for $24 \mathrm{~h}$.

\section{Microinjection}

The intra-accumbens microinjections were given to handrestrained, conscious animals. The stylets were withdrawn from the guide cannulae and injection needles (30 gauge) were inserted, extending $2.5 \mathrm{~mm}$ from the tip of the guide cannulae. The injection needles were connected via polyethylene tubing to two
$10 \mu \mathrm{L}$ Hamilton micro syringes, driven by an automated microinfusion pump (Carnegie Medicine). A total volume of $1 \mu \mathrm{L}$ and $0.5 \mu \mathrm{L}$ per hemisphere were delivered in the NAcc shell and core respectively at a rate of $0.5 \mu \mathrm{L} / \mathrm{min}$ in both cases. After the injections, the needles were retained in the guide cannulae for an additional $1 \mathrm{~min}$ to allow diffusion of the solution into the tissue minimizing the reflux along the injection track. We decided to use a differential volume in each of the NAcc subnuclei since there are reports using a volume of $1 \mu \mathrm{L}$ in the NAcc shell (Sederholm et al. 2002) and 0.5 $\mu \mathrm{L}$ into the NAcc core (Di Ciano et al. 2001). Since the concentration of the drug remained constant for both subnuclei, the amount of drug molecules per volume remained constant, growing larger as the volume increased in the NAcc shell.

\section{Chemicals and drugs}

ACSF solution $(118 \mathrm{mM} \mathrm{NaCl}, 19 \mathrm{mM} \mathrm{NaHCO}, 4.7 \mathrm{mM} \mathrm{KCl}$, $1.2 \mathrm{mM} \mathrm{KH}_{2} \mathrm{PO}_{4}, 1.2 \mathrm{mM} \mathrm{MgSO}_{4}$, and $2.5 \mathrm{mM} \mathrm{CaCl}_{2}$, pH 7.4; T.J. Baker; $3.3 \mathrm{mM}$ glucose, Sigma) was used as vehicle. The NMDA receptor antagonist, DL-2-amino-5-phosphonovaleric acid (APV; $38 \mathrm{mM}, \mathrm{RBI} ; 3.5 \mu \mathrm{g} / 0.5 \mu \mathrm{L}$ ), and the muscarinic receptor antagonist, scopolamine hydrobromide $(136 \mathrm{mM}$, Sigma; $30 \mu \mathrm{g} / 0.5 \mu \mathrm{L})$ were dissolved in ACSF solution. Although a dose of $50.7 \mathrm{mM}$ of DL-APV, has already been used in NAcc during instrumental learning (Giertler et al. 2005), we decided to use a dose of $38 \mathrm{mM}$ of DL-APV, since a preliminary study using $50.7 \mathrm{mM}$ showed that various animals presented motor abnormalities. The dose of scopolamine chosen was the same dose used in other brain structures like insular cortex during the taste memory formation (Naor and Dudai 1996; Ramírez-Lugo et al. 2003).

\section{Behavioral procedures}

In order to determine the possible early and/or late involvement of glutamatergic and cholinergic systems on both the NAcc shell and core in aversive TMT (CTA), APV or scopolamine were administered either 15 min before or immediately after the CS (saccharine) presentation during acquisition. Short-Term Memory (STM) and Long-Term Memory (LTM) were evaluated as described below. Rats received tap water for 15 min just after saccharine presentation in STM and LTM tests.

\section{Conditioned taste aversion (CTA)}

The behavioral procedure for CTA was made as described previously (Ferreira et al. 2002). From d 1 to d 4, rats were trained to take a water ration twice a day in their home cages in order to test STM. Each morning, subjects had access to $10 \mathrm{~mL}$ of tap water during a 15 -min period; then $4.5 \mathrm{~h}$ later, they had access to 40 $\mathrm{mL}$ of water for $15 \mathrm{~min}$. During each presentation, water was given in graduated test tubes, which allowed consumption to be measured.

On d 5, the acquisition day, rats were divided into different groups (see below), and subjects were allowed to drink $10 \mathrm{~mL}$ of a novel taste solution ( $0.1 \%$ saccharine; Sigma) the same volume of water to which the rats had access at the same hour in previous days. Fifteen minutes after the drinking session, a malaiseinducing drug (Lithium Chloride, LiCl, $0.4 \mathrm{M}, 7.5 \mathrm{~mL} / \mathrm{kg}$; Baker) was administered intraperitoneally. Four hours after the LiCl injection, rats were exposed to saccharine solution for $15 \mathrm{~min}$ to assess the STM of CTA (see Figs. 2A, 3A).

During the next $2 \mathrm{~d}$, baseline intake of tap water was reestablished. Three days after acquisition and STM, all rats were exposed to saccharine solution for $15 \mathrm{~min}$ to assess the LTM of CTA. In both STM and LTM tests, reduction of liquid consumption with respect to baseline water intake (average of the first $4 \mathrm{~d}$ of water intake), was used as a measure of strength of aversion: percentage of baseline consumption $=100 \times$ (saccharine solution intake/mean of baseline water consumption).

The animals with cannulae in the NAcc shell were divided into six groups. The groups that received a drug injection before saccharine presentation: Veh-pre $(\mathrm{n}=12)$, APV-pre $(\mathrm{n}=14)$, Scop-pre $(\mathrm{n}=8)$, and the groups that received a drug injection after saccharine presentations (see Fig. 3A): Veh-post $(n=12)$, 
APV-post $(n=8)$ and Scop-post $(n=6)$. The animals with cannulae in the NAcc core were divided into six groups. The groups that received a drug injection before saccharine presentation Veh-pre $(n=9)$, APV-pre $(n=8)$, Scop-pre $(n=6)$, and the groups that received a drug injection after saccharine presentation (see Fig. 3A): Veh-post $(n=5)$, APV-post $(n=6)$ and Scop-post $(n=7)$.

\section{Neophobia and attenuation of neophobia (AN)}

In order to test if APV or scopolamine affect taste perception or safe taste memory, we injected these drugs into the NAcc shell and core before presenting a strong concentration of saccharine $(0.5 \%)$ in the drinking water, and measured the neophobic response, consisting of a lower water intake when it contains saccharine, as compared to plain water consumption. We also measured the safe TMT (attenuation of neophobia), which consists of an increase in saccharine consumption in presentations that follow the initial aversive one.

The behavioral procedure for AN was made as described previously (Gutierrez et al. 2003a). To determine the basal water consumption (baseline water intake) rats were given water in their home cages for $15 \mathrm{~min}$ and volume consumption was registered. This was done every $23.5 \mathrm{~h}$ for $4 \mathrm{~d}$. On day 5, after assigning the animals to different groups (see below) counterbalanced by basal water consumption, the neophobic response was tested by the presentation of $0.5 \%$ saccharine (Sigma) in the drinking water for $15 \mathrm{~min}$ (see Fig. 4A). Thereafter the rats received water access for $15 \mathrm{~min}$ in order to ensure that all animals consumed their daily fluid requirement regardless of their consumption of saccharine. The same procedure was repeated from d 5-8 for AN protocol. The neophobic response was analyzed in terms of a lower intake of the novel taste solution compared to baseline intake of water. AN was then observed as an increased consumption of saccharine solution in the following presentations. The neophobic response and AN are expressed as a percentage of baseline consumption $=100 \times$ (saccharine solution intake/mean baseline water consumption). The drugs were administrated before the taste presentation. We divided the animals into three groups for each, NAcc shell: Veh-pre $(n=16)$, APV-pre $(n=7)$ and Scop-pre $(n=13)$. NAcc core: Veh-pre $(n=6)$, APV-pre $(n=6)$ and Scop-pre $(n=5)$.

\section{Histology}

After completion of the behavioral experiments, animals were overdosed with sodium pentobarbital, and perfused with $0.9 \%$ saline followed by a buffered $4 \%$ paraformaldehyde solution. The brains were removed and stored at $4-10^{\circ} \mathrm{C}$ in the same paraformaldehyde solution during $24 \mathrm{~h}$. The brains were then put in a $30 \%$ glucose solution. They were cut on a freezing cryostat in $40 \mu \mathrm{m}$ slices. Mounted samples were stained with cresyl violet and then examined in a light microscope. Slices were examined under the microscope to determine the correct placement of the cannulae in the brain structures.

\section{Acknowledgments}

This work was supported by CONACyT 42657Q and DGAPAUNAM IX202504-1. We acknowledge the technical assistance of Federico Jandete, Oreste Carbajal, Francisco Pérez Eugenio, Enrique Espinosa, and Adriana Vega Vega for their text review and give thanks to Luis Nuñez Jaramillo for his comments on this manuscript.

\section{References}

Bermúdez-Rattoni, F. 2004. Molecular mechanisms of taste-recognition memory. Nat. Rev. Neurosci. 5: 209-217.

Berridge, K.C. and Robinson, T.E. 1998. What is the role of dopamine in reward: Hedonic impact, reward learning, or incentive salience? Brain Res. Brain Res. Rev. 28: 309-369.

Bliss, T.V. and Collingridge, G.L. 1993. A synaptic model of memory: Long-term potentiation in the hippocampus. Nature 361: 31-39.

Bolam, J.P., Wainer, B.H., and Smith, A.D. 1984. Characterization of cholinergic neurons in the rat neostriatum. A combination of choline acetyltransferase immunocytochemistry, Golgi-impregnation and electron microscopy. Neuroscience 12: 711-718.

Brog, J.S., Salyapongse, A., Deutch, A.Y. and Zahm, D.S. 1993. The patterns of afferent innervation of the core and shell in the "accumbens" part of the rat ventral striatum: Immunohistochemical detection of retrogradely transported fluoro-gold. J. Comp. Neurol. 338: $255-278$.

Centonze, D., Gubellini, P., Bernardi, G., and Calabresi, P. 1999. Permissive role of interneurons in corticostriatal synaptic plasticity. Brain Res. Brain Res. Rev. 31: 1-5.

Centonze, D., Gubellini, P., Pisani, A., Bernardi, G. and Calabresi, P. 2003. Dopamine, acetylcholine and nitric oxide systems interact to induce corticostriatal synaptic plasticity. Rev. Neurosci. 14: 207-216.

De Leonibus, E., Costantini, V.J., Castellano, C., Ferretti, V., Oliverio, A., and Mele, A. 2003. Distinct roles of the different ionotropic glutamate receptors within the nucleus accumbens in passive-avoidance learning and memory in mice. Eur. J. Neurosci. 18: $2365-2373$.

Delfs, J.M., Zhu, Y., Druhan, J.P., and Aston-Jones, G.S. 1998. Origin of noradrenergic afferents to the shell subregion of the nucleus accumbens: Anterograde and retrograde tract-tracing studies in the rat. Brain Res. 806: $127-140$.

de Rover, M., Lodder, J.C., Kits, K.S., Schoffelmeer, A.N., and Brussaard, A.B. 2002. Cholinergic modulation of nucleus accumbens medium spiny neurons. Eur. J. Neurosci. 16: 2279-2290.

Di Ciano, P., Cardinal, R.N., Cowell, R.A., Little, S.J., and Everitt, B.J. 2001. Differential involvement of NMDA, AMPA/kainate, and dopamine receptors in the nucleus accumbens core in the acquisition and performance of Pavlovian approach behavior. J. Neurosci. 21: 9471-9477.

Everitt, B.J., Morris, K.A., O’Brien, A., and Robbins, T.W. 1991. The basolateral amygdala-ventral striatal system and conditioned place preference: Further evidence of limbic-striatal interactions underlying reward-related processes. Neuroscience 42: 1-18.

Everitt, B.J., Parkinson, J.A., Olmstead, M.C., Arroyo, M., Robledo, P., and Robbins, T.W. 1999. Associative processes in addiction and reward. The role of amygdala-ventral striatal subsystems. Ann. N.Y. Acad. Sci. 877: 412-438.

Fenu, S., Bassareo, V., and Di Chiara, G. 2001. A role for dopamine D1 receptors of the nucleus accumbens shell in conditioned taste aversion learning. J. Neurosci. 21: 6897-6904.

Ferreira, G., Gutierrez, R., De La Cruz, V., and Bermúdez-Rattoni, F. 2002. Differential involvement of cortical muscarinic and NMDA receptors in short- and long-term taste aversion memory. Eur. J. Neurosci. 16: 1139-1145.

Giertler, C., Bohn, I., and Hauber, W. 2005. Involvement of NMDA and AMPA/KA receptors in the nucleus accumbens core in instrumental learning guided by reward-predictive cues. Eur. J. Neurosci. 21: $1689-1702$.

Gottfried, J.A., O’Doherty, J., and Dolan, R.J. 2002. Appetitive and aversive olfactory learning in humans studied using event-related functional magnetic resonance imaging. J. Neurosci. 22: 1082910837.

Gutierrez, R., Rodriguez-Ortiz, C.J., De La Cruz, V., Nunez-Jaramillo, L., and Bermúdez-Rattoni, F. 2003a. Cholinergic dependence of taste memory formation: Evidence of two distinct processes. Neurobiol. Learn. Mem. 80: 323-331.

Gutierrez, R., Tellez, L.A., and Bermúdez-Rattoni, F. 2003b. Blockade of cortical muscarinic but not NMDA receptors prevents a novel taste from becoming familiar. Eur. J. Neurosci. 17: 1556-1562.

Jasmin, L., Granato, A., and Ohara, P.T. 2004. Rostral agranular insular cortex and pain areas of the central nervous system: A tract-tracing study in the rat. J. Comp. Neurol. 468: 425-440.

Kelley, A.E. and Domesick, V.B. 1982. The distribution of the projection from the hippocampal formation to the nucleus accumbens in the rat: An anterograde- and retrograde-horseradish peroxidase study. Neuroscience 7: 2321-2335.

Kelley, A.E. and Swanson, C.J. 1997. Feeding induced by blockade of AMPA and kainate receptors within the ventral striatum: A microinfusion mapping study. Behav. Brain Res. 89: 107-113.

Kelley, A.E., Smith-Roe, S.L., and Holahan, M.R. 1997. Response-reinforcement learning is dependent on $\mathrm{N}$-methyl-D-aspartate receptor activation in the nucleus accumbens core. Proc. Natl. Acad. Sci. 94: 12174-12179.

Louilot, A. and Besson, C. 2000. Specificity of amygdalostriatal interactions in the involvement of mesencephalic dopaminergic neurons in affective perception. Neuroscience 96: 73-82.

Mark, G.P., Blander, D.S., and Hoebel, B.G. 1991. A conditioned stimulus decreases extracellular dopamine in the nucleus accumbens after the development of a learned taste aversion. Brain Res. 551: 308-310.

Mark, G.P., Weinberg, J.B., Rada, P.V., and Hoebel, B.G. 1995.

\section{Learning \& Memory}


Extracellular acetylcholine is increased in the nucleus accumbens following the presentation of an aversively conditioned taste stimulus. Brain Res. 688: 184-188.

Martinez, G., Ropero, C., Funes, A., Flores, E., Landa, A.I., and Gargiulo, P.A. 2002. AP-7 into the nucleus accumbens disrupts acquisition but does not affect consolidation in a passive avoidance task. Physiol. Behav. 76: 205-212.

McDonald, A.J. 1991. Organization of amygdaloid projections to the prefrontal cortex and associated striatum in the rat. Neuroscience 44: $1-14$.

Mehendale, S., Xie, J.T., Aung, H.H., Guan, X.F., and Yuan, C.S. 2004. Nucleus accumbens receives gastric vagal inputs. Acta. Pharmacol. Sin. 25: 271-275.

Meredith, G.E. 1999. The synaptic framework for chemical signaling in nucleus accumbens. Ann. N.Y. Acad. Sci. 877: 140-156.

Meredith, G.E., Blank, B., and Groenewegen, H.J. 1989. The distribution and compartmental organization of the cholinergic neurons in nucleus accumbens of the rat. Neuroscience 31: 327-345.

Miranda, M.I. and Bermúdez-Rattoni, F. 1999. Reversible inactivation of the nucleus basalis magnocellularis induces disruption of cortical acetylcholine release and acquisition, but not retrieval, of aversive memories. Proc. Natl. Acad. Sci. 96: 6478-6482.

Miranda, M.I., Ferreira, G., Ramírez-Lugo, L., and Bermúdez-Rattoni, F. 2002. Glutamatergic activity in the amygdala signals visceral input during taste memory formation. Proc. Natl. Acad. Sci. 99: 1141711422 .

Naor, C. and Dudai, Y. 1996. Transient impairment of cholinergic function in the rat insular cortex disrupts the encoding of taste in conditioned taste aversion. Behav. Brain Res. 79: 61-67.

Neigh, G.N., Arnold, H.M., Rabenstein, R.L., Sarter, M., and Bruno, J.P. 2004. Neuronal activity in the nucleus accumbens is necessary for performance-related increases in cortical acetylcholine release. Neuroscience 123: 635-645.

Neigh-McCandless, G., Kravitz, B.A., Sarter, M., and Bruno, J.P. 2002. Stimulation of cortical acetylcholine release following blockade of ionotropic glutamate receptors in nucleus accumbens. Eur. J. Neurosci. 16: 1259-1266.

Ohara, P.T., Granato, A., Moallem, T.M., Wang, B.R., Tillet, Y., and Jasmin, L. 2003. Dopaminergic input to GABAergic neurons in the rostral agranular insular cortex of the rat. J. Neurocytol. 32: 131-141.

Paxinos, G. and Watson, C. 1998. The rat brain in stereotaxic coordinates. Academic Press, San Diego, California.

Pennartz, C.M., Groenewegen, H.J., and Lopes da Silva, F.H. 1994. The nucleus accumbens as a complex of functionally distinct neuronal ensembles: An integration of behavioural, electrophysiological and anatomical data. Prog. Neurobiol. 42: 719-761.

Pezze, M.A., Heidbreder, C.A., Feldon, J., and Murphy, C.A. 2001. Selective responding of nucleus accumbens core and shell dopamine to aversively conditioned contextual and discrete stimuli.

Neuroscience 108: 91-102.
Ramírez-Lugo, L., Miranda, M.I., Escobar, M.L., Espinosa, E., and Bermúdez-Rattoni, F. 2003. The role of cortical cholinergic pre- and post-synaptic receptors in taste memory formation. Neurobiol. Learn. Mem. 79: 184-193.

Salamone, J.D. 1994. The involvement of nucleus accumbens dopamine in appetitive and aversive motivation. Behav. Brain Res. 61: 117-133.

Salamone, J.D. 1996. The behavioral neurochemistry of motivation: Methodological and conceptual issues in studies of the dynamic activity of nucleus accumbens dopamine. J. Neurosci. Methods 64: $137-149$.

Salamone, J.D., Correa, M., Mingote, S.M., and Weber, S.M. 2005. Beyond the reward hypothesis: Alternative functions of nucleus accumbens dopamine. Curr. Opin. Pharmacol. 5: 34-41.

Saul'skaya, N.B. and Mikhailova, M.O. 2003. The effects of motivational and emotional factors in glutamate release in the nucleus accumbens of the rat brain during food consumption. Neurosci. Behav. Physiol. 33: 151-156.

Sederholm, F., Johnson, A.E., Brodin, U., and Sodersten, P. 2002. Dopamine $\mathrm{D}(2)$ receptors and ingestive behavior: Brainstem mediates inhibition of intraoral intake and accumbens mediates aversive taste behavior in male rats. Psychopharmacology (Berl). 160: 161-169.

Swanson, L.W. and Petrovich, G.D. 1998. What is the amygdala? Trends Neurosci. 21: 323-331.

Wright, C.I. and Groenewegen, H.J. 1996. Patterns of overlap and segregation between insular cortical, intermediodorsal thalamic and basal amygdaloid afferents in the nucleus accumbens of the rat. Neuroscience 73: 359-373.

Yamamoto, T., Shimura, T., Sako, N., Yasoshima, Y., and Sakai, N. 1994. Neural substrates for conditioned taste aversion in the rat. Behav. Brain Res. 65: 123-137.

Young, A.M. 2004. Increased extracellular dopamine in nucleus accumbens in response to unconditioned and conditioned aversive stimuli: Studies using 1 min microdialysis in rats. J. Neurosci. Methods 138: $57-63$.

Young, A.M., Joseph, M.H., and Gray, J.A. 1993. Latent inhibition of conditioned dopamine release in rat nucleus accumbens. Neuroscience 54: 5-9.

Zahm, D.S. 2000. An integrative neuroanatomical perspective on some subcortical substrates of adaptive responding with emphasis on the nucleus accumbens. Neurosci. Biobehav. Rev. 24: 85-105.

Zahm, D.S. and Heimer, L. 1993. Specificity in the efferent projections of the nucleus accumbens in the rat: Comparison of the rostral pole projection patterns with those of the core and shell. J. Comp. Neurol. 327: 220-232.

Received September 30, 2005; accepted in revised form December 1, 2005. 


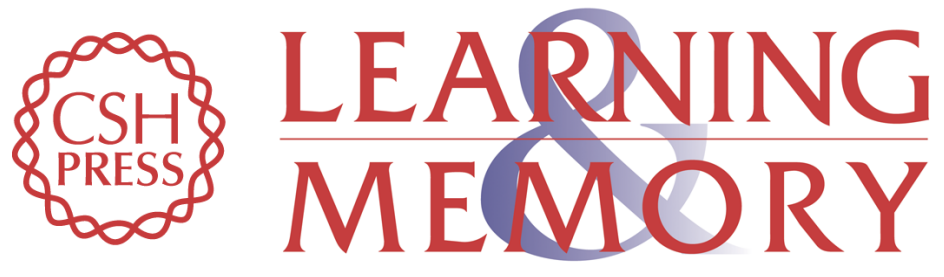

\section{NMDA and muscarinic receptors of the nucleus accumbens have differential effects on taste memory formation}

Leticia Ramírez-Lugo, Sergio Zavala-Vega and Federico Bermúdez-Rattoni

Learn. Mem. 2006, 13:

Access the most recent version at doi:10.1101//m.103206

References This article cites 52 articles, 6 of which can be accessed free at:

http://learnmem.cshlp.org/content/13/1/45.full.html\#ref-list-1

License

Email Alerting Receive free email alerts when new articles cite this article - sign up in the box at the Service top right corner of the article or click here. 